\title{
THE NIGHTSIDE IONOSPHERE OF VENUS UNDER VARYING LEVELS OF SOLAR EUV FLUX
}

\author{
C. M. Ho†, R. J. Strangeway, C. T. Russell, and J. G. Luhmann \\ Institute of Geophysics and Planetary Physics, University of California
}

L. H. Brace

Space Physics Research Laboratory, University of Michigan

\begin{abstract}
Solar activity varied widely over the 14 year lifetime of the Pioneer Venus Orbiter, and these variations directly affected the properties of the nightside ionosphere. At solar maximum, when solar EUV was largest, the Venus ionosphere was found to extend to highest altitudes and nightward ion transport was the main source of the nightside ionosphere. At solar minimum, nightward ion transport was reduced, and electron precipitation was thought to be the main source. In this study, we have attempted a separation of spatial variations from temporal variations by examining the altitude profiles of the magnetic field, and electron density and temperature for three different solar EUV flux ranges. In the upper ionosphere and near-planet magnetotail $(h>1800$ $\mathrm{km}$ ), the solar EUV effects are significant. The electron density decreases about an order of magnitude from high to low EUV flux, while the electron temperature at least doubles. The magnetic field also increases $2-3 \mathrm{nT}$. In the lower ionosphere $(200-600 \mathrm{~km})$, lower EUV fluxes are associated with slightly reduced density, and higher temperature. These results are in accord with recent entry phase observations, where the electron density measured above the ionospheric density peak is lower than that observed at solar maximum during the early Pioneer Venus mission.
\end{abstract}

\section{Introduction}

The entry of the Pioneer Venus Orbiter (PVO) into the low altitude Venus ionosphere occurred at a time of intermediate solar activity, when the nightside ionosphere was likely to be different from the ionosphere observed early in the mission. The earlier measurements of the PVO at solar maximum revealed that there is a surprisingly large nightside ionosphere on Venus [Brace et al., 1987]. This ionosphere is produced by a combination of ion flow from the dayside and local ion production by energetic electron impact. The absence of a planetary magnetic field permits this nightward flow, which seems to have a relative importance that varies with solar activity (e.g. Knudsen et al. [1987]). Larger flows occur at solar maximum when the dayside density is high, while local production may be more important than transport at solar minimum [Gringauz et al., 1979; Brace et al., 1990] when the amount of nightward flow is reduced due to lower-

†Now at Jet Propulsion Laboratory

Copyright 1993 by the American Geophysical Union. ing of the ionopause altitude. The solar cycle variation in the nightside ionosphere is likely to be larger than in the dayside because of the large changes in the nightward ion flux that helps maintain the nightside ionosphere.

Knudsen [1988] used ion measurements to infer the presence of a strong solar cycle variation in the central nightside ionosphere up to $2000 \mathrm{~km}$. Knudsen found a significant discontinuity in the median density altitude profiles from the high altitude near-solar minimum and the low altitude near-solar maximum data. He concluded that this apparent jump in density with altitude between the two different periods was due to a solar cycle variation, rather than a ledge in the ion density at that altitude. In his study there was no overlap in the coverage at any altitude for low and high solar activity. This is because significant changes in the PVO periapsis altitude occurred during the 14 year lifetime of the mission, roughly in anti-phase with the solar cycle.

In this study we analyze the effect of solar EUV on the nightside ionospheric structure in more detail than in previous studies, using data from 1979 to 1987 . This complements the entry phase studies of Theis and Brace [1993] and Russell et al. [1993]. Because the EUV flux is expected to have an immediate effect on the plasma density of the dayside ionosphere and the nightward plasma flow, we use the daily EUV level (the $V_{\text {EUV }}$ index) rather than the seasonal average in characterizing the observations. The $\mathrm{V}_{\mathrm{EUV}}$ index was derived by Brace et al. [1988] from the photoelectron emission current resulting from EUV illumination of the PVO Langmuir probe. These values vary significantly from day to day as the Sun rotates, especially at solar maximum. Thus it is possible to encounter a range of EUV activity at any altitude, allowing us to determine the altitude dependence at different levels of EUV flux. In this study, however, we have not made any distinction between short-term ( 28 day) and long-term (solar cycle) EUV variations. This distinction may be important at the lowest altitudes, where the coupling between the neutral atmosphere and ionosphere is greatest [Brace et al., 1990]. On the other hand, the high altitude ionosphere is more likely to respond to both solar cycle and solar rotation related variations in EUV flux.

Observations show that the electron density of the nearplanet magnetotail is greatly depleted at solar minimum. The density decreases by a factor of 20 or more for a $30 \%$ decrease of the solar EUV flux [Brace et al., 1990]. This variation also may affect the magnetotail magnetic field through changes in the nightward plasma flow. However, the solar cycle variations of the magnetic field, if present, have not been reported [Luhmann and Cravens, 1991]. In addition, the dependence of the altitudinal structure of the nightside ionosphere on changes of EUV flux remains unknown. 


\section{Solar EUV Flux at Venus}

Figure 1 shows the solar EUV flux at Venus from 1979 to 1987 as measured once per day by the PVO Langmuir Probe [Brace et al., 1988]. The photo-electron current due to EUV can be measured by the Langmuir probe when the Orbiter is in the solar wind, where the electron density is low. Brace et al. [1988] have determined a scaling law relating photo-electron current to EUV flux. Figure 1 shows that the EUV flux decreased by a factor of two from solar maximum in 1979 to solar minimum in 1987.

Figure 1 also shows the PVO nightside periapsis altitude for the same period. Direct measurements of the lower ionosphere were obtained only during the solar maximum condition of $1979-1981$. The subsequent rise in periapsis made it impossible to observe the response of the main ionosphere to the decline of solar activity. When the solar activity reached intermediate levels in 1982, periapsis had increased to an altitude of $1000 \mathrm{~km}$. The observations of the near-planet magnetotail $(1800-3000 \mathrm{~km})$ were all made near solar minimum (1984 - 1987). They are thus not representative of conditions at high levels of solar activity.

To distinguish between spatial variations of the ionosphere and those due to changes of solar activity we divide the data into three levels of solar EUV flux. We define high EUV fluxes as greater than $14.5 \times 10^{11}$ photon $/ \mathrm{cm}^{2} / \mathrm{sec}$. Intermediate EUV fluxes are in the range $11.5 \times 10^{11}$ photon $/ \mathrm{cm}^{2} / \mathrm{sec}$ to $14.5 \times 10^{11}$ photon $/ \mathrm{cm}^{2} / \mathrm{sec}$, and low EUV fluxes are below $11.5 \times 10^{11}$ photon $/ \mathrm{cm}^{2} / \mathrm{sec}$. The solar EUV flux has large fluctuations during solar maximum, and we can examine a range of EUV conditions at a fixed altitude. However, these fluctuations are mainly caused by slowly varying solar sources that rotate by the spacecraft every 28 days. Figure 1 shows that at low altitudes $(<1500 \mathrm{~km})$ most of the variation in EUV flux is due to solar rotation, while at high altitudes ( $>1500 \mathrm{~km}$ ) the variation is mainly due to the secular change over the solar cycle.

\section{Results}

We have examined all nightside magnetic field and electron data from 1979 to 1987 using 12-s averaged data from

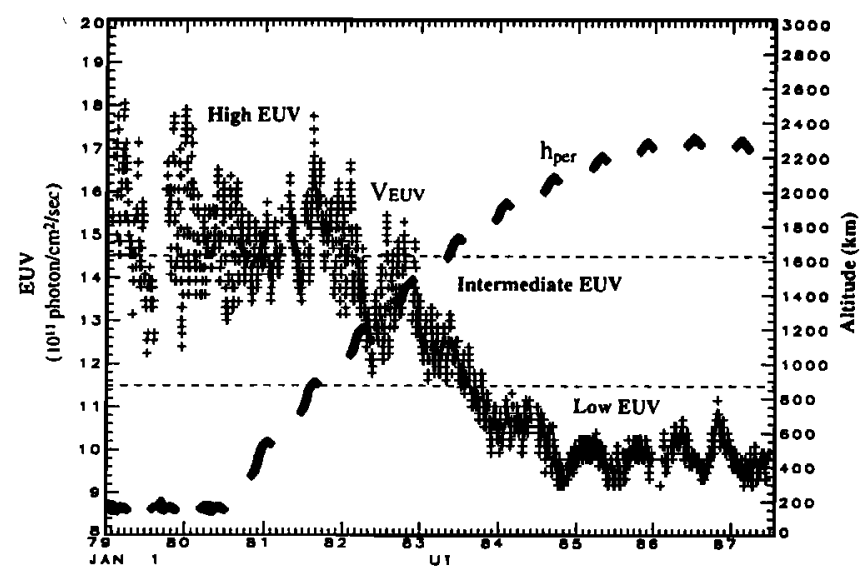

Fig. 1. Solar EUV flux (VEV) as measured by the PVO Langmuir probe from 1979 (solar maximum) to 1987 (solar minimum). The PVO nightside periapsis altitude $\left(\mathrm{h}_{\mathrm{per}}\right)$ is also shown. Solar EUV flux is divided into three activity ranges for this study.

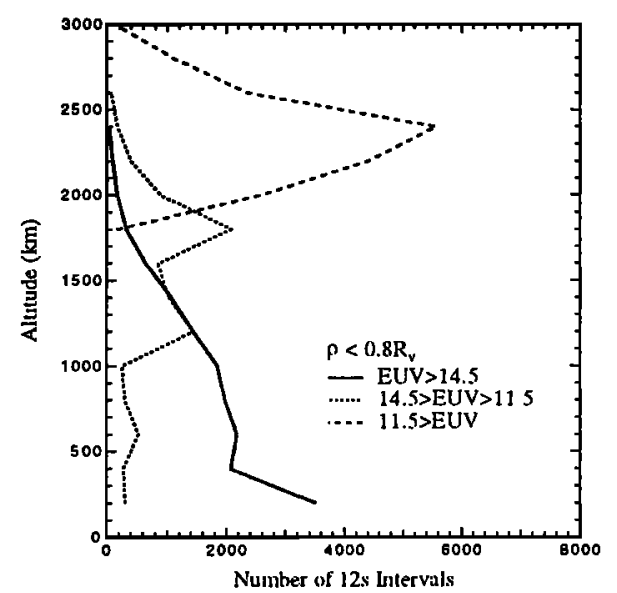

Fig. 2. Magnetic field data coverage for three different EUV flux levels. High (solid line), intermediate (dotted line) and low (dashed line) EUV fluxes are shown. The data have been restricted to $\rho<0.8 R_{v}$, inside the optical shadow.

the Unified Abstract Data System (UADS). The data coverage for magnetic field measurements for various EUV fluxes is shown in Figure 2 as a function of altitude, where we have binned the data into $200 \mathrm{~km}$ altitude bins. The Langmuir probe data tend to be sampled less frequently, especially at higher altitudes, but this sampling is reflected in the error bars in subsequent plots. The three EUV flux levels that we chose to use here have different altitude coverage. There are 15,411 12-s intervals at high EUV fluxes (solid line). They mainly cover altitudes below $2000 \mathrm{~km}$. There are fewer data at intermediate EUV fluxes (dotted line) (8,654 12-s intervals), but they cover almost all altitudes. There are more data (16,332 12-s intervals) at low EUV fluxes (dashed line), but these are all found at high altitudes above $1800 \mathrm{~km}$. However, there is some overlap between high and intermediate EUV flux from $600 \mathrm{~km}$ to $1800 \mathrm{~km}$ altitude, and between intermediate and low EUV flux from $1800 \mathrm{~km}$ to $2400 \mathrm{~km}$ altitude.

The altitude profiles of the electron density $\left(n_{e}\right)$, temperature $\left(T_{e}\right)$ and total magnetic field $\left(B_{t}\right)$ for the different EUV flux levels are shown in Figures 3,4 and 5, again using $200 \mathrm{~km}$ altitude bins. In each profile we also give the standard error of the mean. The variations of the electron density

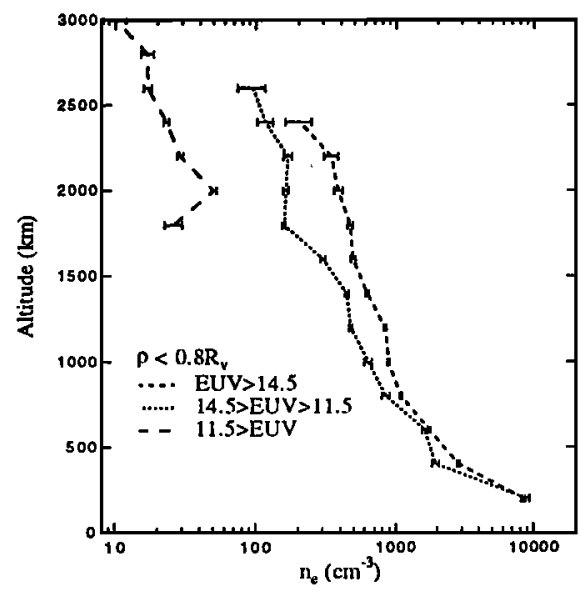

Fig. 3. Altitude profiles of the electron density in the nightside ionosphere for different EUV flux levels. The standard error of the mean is shown as error bars on each profile. 


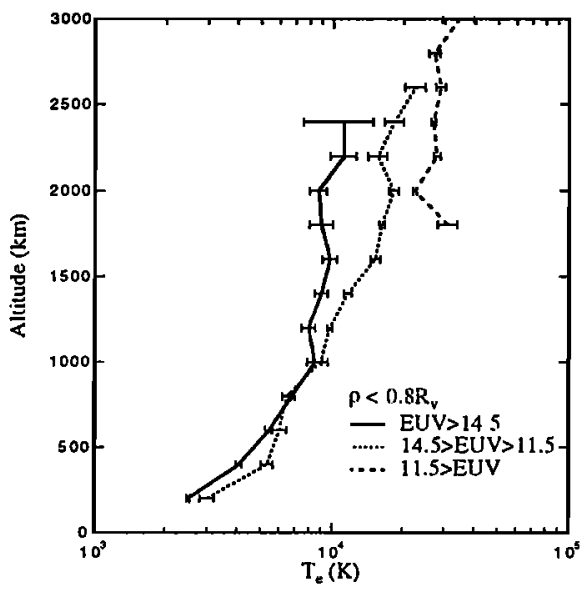

Fig. 4. The electron temperature variation in the nightside ionosphere for different EUV flux levels.

clearly show the existence of a variation with EUV flux level. The average $n_{e}$ has a peak value about $10^{4} \mathrm{~cm}^{-3}$ in the lowest altitude bin, centered on $200 \mathrm{~km}$, with the density gradually decreasing with increasing altitude. In the upper ionosphere the density falls to around $300 \mathrm{~cm}^{-3}$ for high EUV flux (solid line). There is a large overlap of altitudes between high EUV flux and intermediate EUV flux (dotted line). The average density is lower for lower EUV flux as expected. This difference becomes most obvious in the upper ionosphere. For low EUV flux (dashed line), the electron density in the near-planet magnetotail drops as much as an order of magnitude, relative to high EUV flux. This change is similar to that reported in the previous study of Brace et al. [1990]. This strong EUV flux control of the nightside electron density is thought to take place through a reduction of the nightward plasma flow [Brace and Kliore, 1991].

In contrast to the electron density dependence, the electron temperature increases with decreasing EUV flux as shown in Figure 4. In the lower ionosphere, the average temperature for the high EUV flux (solid line) is slightly lower than that for intermediate EUV flux (dotted line). The electron temperature gradually increases from $2.5 \times 10^{3} \mathrm{~K}$ in the $200 \mathrm{~km}$ bin to $\sim 10^{4} \mathrm{~K}$ in the upper ionosphere. For low solar EUV flux (dashed line), the temperature jumps to near $3 \times 10^{4} \mathrm{~K}$. Thus the variation of the electron temperature is

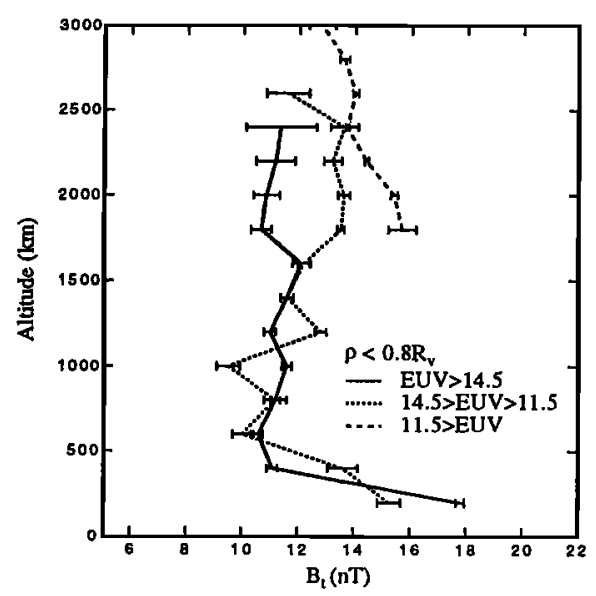

Fig. 5. Altitude dependence of the magnetic field in the nightside ionosphere for different EUV flux levels. basically the inverse of the variation of the electron density. In the near tail the temperature changes by a factor of $2-3$ from high to low EUV fluxes.

The altitude dependence of the magnetic field for different EUV fluxes is shown in Figure 5. For high EUV flux (solid line) the average $B_{t}$ has a maximum value about $18 \mathrm{nT}$ at lowest altitude $(\sim 200 \mathrm{~km})$. This $B_{t}$ peak is similar to those large-scale field structures observed in the lower dayside ionosphere [Luhmann and Cravens, 1991]. The field quickly decreases with increasing altitude. At $600 \mathrm{~km}$ the field reaches about $10 \mathrm{nT}$ and then slightly increases to $12 \mathrm{nT}$ at higher altitude. Above $1600 \mathrm{~km}$ the field decreases again, but with increasing uncertainty. Most of the change in the magnetic field with altitude therefore occurs at lowest altitude for high EUV flux. In the lower ionosphere the average field is roughly the same for intermediate EUV flux (dotted line) as for high EUV flux, but with larger uncertainty. Above $1600 \mathrm{~km}$ the magnetic field increases $2-3 \mathrm{nT}$ for both intermediate and low EUV flux (dashed line), relative to high EUV flux. There is also a significant decrease in $n_{e}$ and an increase in $T_{e}$ in this altitude range. The increase of $B_{t}$ possibly arises to maintain pressure balance with the magnetosheath. Because plasma dynamic pressure is unimportant in the upper ionosphere, the magnetic pressure will be anti-correlated with the plasma thermal pressure.

We have examined the local time variation of the magnetic field and the electron density in the low ionosphere above the density peak $(200 \mathrm{~km}<\mathrm{h}<600 \mathrm{~km})$ during high and intermediate EUV conditions, as shown in Figure 6. There is some asymmetry about midnight in these distributions. From the upper panel we see that for high EUV flux $n_{e}$ decreases about a factor of 4 from both dawn and dusk to midnight. For intermediate EUV flux this decrease may be as large as an order of magnitude. At most local times the nightside electron density is lower for intermediate EUV flux than for high EUV flux, but with relatively large fluctuations. This variation may be partially due to fewer data samples for lower EUV fluxes. However, the low altitude ionosphere ( $>200 \mathrm{~km}$ ) has consistently lower electron den-

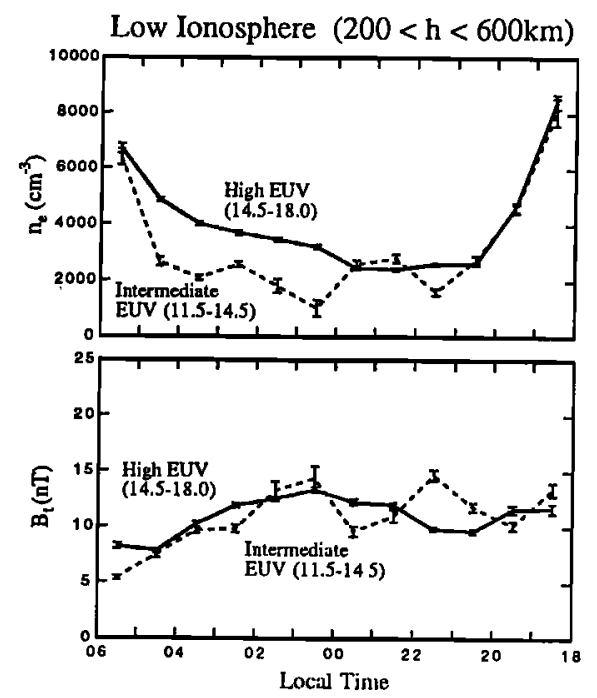

Fig. 6. Local time dependence of the magnetic field and electron density in the lower ionosphere for two different EUV flux levels. The average values for each hour are obtained using a two-hour window. 
sity for intermediate EUV conditions in the post-midnight local time sector, where PVO atmospheric entry occurred. The magnetic field is generally anti-correlated with electron density. The field strength is larger around midnight and the dusk terminator, compared to dawn.

\section{Summary}

Although PVO observations were obtained for more than an entire solar cycle, changes in the periapsis altitude make it difficult to separate the altitudinal structure from the temporal or solar cycle variation. The evolution of the PVO orbit has allowed the main nightside ionosphere to be examined only during solar maximum, while the measurements of the upper ionosphere mainly were made near solar minimum. We have used statistical results to show that apparent discontinuities in the altitude profiles are caused by a variation of the solar EUV flux and are not due to a true altitude variation.

In the upper ionosphere above $1800 \mathrm{~km}$, there are some significant changes for the plasma and magnetic field for different solar EUV conditions. For low EUV flux levels, the electron density decreases about an order of magnitude, relative to high EUV flux. Accompanying this change, the electron temperature doubles, while the magnetic field is enhanced by about $2-3 \mathrm{nT}$. These variations are consistent with the weakening of ion transport from dayside to nightside due to the decline of the dayside ionosphere density and terminator ionopause altitude for lower EUV fluxes. The associated depression in the electron density should be accompanied by an increase of magnetic field and electron temperature to maintain pressure balance with the magnetosheath. Both are expected to increase since there is likely to be greater magnetic field transport and heat flux from the magnetosheath when the ionosphere is depleted [Theis and Brace, 1993].

At lower altitudes the solar EUV effect is somewhat smaller. Theis and Brace [1993] show that the electron density peak is essentially the same during the entry phase, compared to the early mission. Using the high and intermediate EUV flux curves as a guide in extrapolating the low EUV flux measurements to altitudes in the range 200 $600 \mathrm{~km}$ (i.e., above the density peak), we expect the average electron density should drop by about a factor of 4 . The electron temperature should slightly increase, while the magnetic field will have no obvious change during solar minimum. This is consistent with the entry phase results of Theis and Brace [1993] and Russell et al. [1993].

Our results suggest that the solar EUV variation strongly controls the structure of the nightside ionosphere. We note that this is a different phenomenon in some sense than the "disappearing ionosphere" [Luhmann and Cravens, 1991] which occurs when transport is cut off during high EUV conditions because of high solar wind dynamic pressure. However, the solar cycle variation of solar wind dynamic pressure will also play some role in controlling the night- ward plasma flow. Thus, the short-term orbit to orbit variations of solar wind dynamic pressure [Brace et al., 1990] probably provide some of the scatter seen in our study.

Acknowledgments. This work was supported by NASA under grants NAG2-485, NAG2-501, NAGW-3492, and NAGW-3497.

\section{References}

Brace, L. H., and A. J. Kliore, The structure of the Venus ionosphere, Space Sci. Rev., 55, 81-163, 1991.

Brace, L. H., W. T. Kasprzak, H. A. Taylor, R. F. Theis, C. T. Russell, A. Barnes, J. D. Mihalov, and D. M. Hunten, The ionotail of Venus: Its configuration and evidence for ion escape, J. Geophys. Res., 92, 15-26, 1987.

Brace, L. H., W. R. Hoegy, R. F. Theis, Solar EUV measurements at Venus based on photoelectron emission from the Pioneer Venus Langmuir probe, J. Geophys. Res., 93, 7282-7296, 1988.

Brace, L. H., R. F. Theis and J. D. Mihalov, Response of nightside ionosphere and ionotail of Venus to variations in solar EUV and solar wind dynamic pressure, $J$. Geophys. Res., 95, 4075-4084, 1990.

Gringauz, K. I., M. I. Verigin, T. K. Breus, and T. Gombosi, The interaction of electrons in the optical umbra of Venus with the planetary atmosphere - the origin of the nighttime ionosphere, J. Geophys. Res., 84, 2123-2127, 1979.

Knudsen, W. C., Solar cycle changes in the morphology of the Venus ionosphere, J. Geophys. Res., 93, 8756-8762, 1988.

Knudsen, W. C., A. J. Kliore, and R. C. Whitten, Solar cycle changes in the ionization sources of the nightside Venus ionosphere, J. Geophys. Res., 92, 13,391-13,398, 1987.

Luhmann, J. G., and T. E. Cravens, Magnetic fields in the ionosphere of Venus, Space Sci. Rev., 55, 201-274, 1991.

Russell, C. T., R. J. Strangeway, J. G. Luhmann and L. H. Brace, The magnetic state of the lower ionosphere during Pioneer Venus entry phase, Geophys. Res. Lett., this issue, 1993.

Theis, R. F., and L. H. Brace, Solar cycle variations of the electron density and temperature in the nightside Venus ionosphere, Geophys. Res. Lett., this issue, 1993.

L. H. Brace, Space Physics Research Laboratory, Department of Atmospheric, Oceanic and Space Science, The University of Michigan, Ann Arbor, MI 48109-2143

C. M. Ho, Jet Propulsion Laboratory, California Institute of Technology, Pasadena, CA 91109-8099

J. G. Luhmann, C. T. Russell, and R. J. Strangeway, Institute of Geophysics and Planetary Physics, University of California at Los Angeles, Los Angeles, CA 90024-1567

(Received: July 27, 1993; accepted: August 20, 1993) 